\title{
A Cone-Dominance Approach for Discrete Alternative Multiple Criteria Problems with Indifference Regions
}

\author{
Vahid Lotfi \\ School of Management, University of Michigan Flint, Flint, USA \\ Email: vahid@umich.edu
}

How to cite this paper: Lotfi, V. (2021) A Cone-Dominance Approach for Discrete Alternative Multiple Criteria Problems with Indifference Regions. Open Access Library Journal, 8: e8093.

https://doi.org/10.4236/oalib.1108093

Received: October 19, 2021

Accepted: November 8, 2021

Published: November 11, 2021

Copyright () 2021 by author(s) and Open Access Library Inc.

This work is licensed under the Creative Commons Attribution International License (CC BY 4.0).

http://creativecommons.org/licenses/by/4.0/

\section{(c) (i) Open Access}

\begin{abstract}
In this paper, we address the problem of solving discrete alternative multiple criteria decision making (MCDM) problems where some or all of the criteria might have indifference regions. We utilize the classical cone-dominance approach and significantly extend the associated theory. We then develop a convergent solution method based on cone-dominance for achieving the most preferred choice. The convergent method utilizes pairwise comparisons of the alternatives by the decision maker (DM) to eliminate inferior or dominated alternatives and to arrive at the optimum. The stated theoretical development significantly strengthens the theory of cones and presents a streamlined approach for solving the stated MCDM problems. We present a numerical example to illustrate the application of the method in finance. We also present a simulation study, evaluating the performance of the method on several hundred randomly generated test problems. Results of the simulation study are analyzed to assess the possible effects of the presence of indifference regions on the required number of pairwise comparisons to reach the optimal choice.
\end{abstract}

\section{Subject Areas}

Discrete Mathematics

\section{Keywords}

Multiple Criteria Decision Making, Discrete Alternative, Cone-Dominance, Indifference Region

\section{Introduction}

In this paper, we present new theoretical developments and associated metho- 
dology for solving discrete alternative multiple criteria decision making (MCDM) problems which include criteria with indifference regions. The problem involves the selection of an alternative, from a finite set of $\mathrm{m}$ decision alternatives with respect to $\mathrm{p}$ criteria or objective. The set $\mathrm{S}$ of alternatives consists of real vectors $z^{k} \in R^{p}, k=1,2, \cdots, m$, that will maximize a decision maker's implicit and unknown value (or utility) function. Each alternative $\mathrm{k}$ is assumed to be deterministic and realizable in terms of the vector $z^{k}=\left(z_{1}^{k}, z_{2}^{k}, \ldots, z_{p}^{k}\right)$ of performance measures on the $\mathrm{p}$ attributes, hence $z_{i}^{k}$ denotes the score for alternative $k$ with respect to objective $i$. The purpose of the decision analysis is to provide a support tool by which the decision maker (DM) can identify a single final ("optimum") alternative from $S$ that will maximize his/her value function [1].

The proposed method is based on utility maximization and uses pairwise comparisons of alternatives. Because the decision maker's value function is unknown, at most $m-1$ pairwise comparisons are required to find the optimal solution. However, the use of cone-dominance has been shown to reduce the number of comparisons [2] [3]. This study significantly extends the earlier works by providing new theoretical developments for discrete alternative MCDM problems that some of the objectives have satisficing or indifference regions. The presence of an indifference region within an objective is rather intuitive as a DM's implicit utility for that criterion might reach a plateau and then level off. To the best of our knowledge, this type of MCDM problem has not been studied before.

Many methods have been suggested for solving the discrete alternative MCDM problems. Ramanathan et al. [4] present an excellent exposition of various types of MCDM problems and associated solution approaches. Most of the existing methods for solving the discrete alternative MCDM problems can be categorized as: a) Multiattribute utility or value maximization approaches [5]-[13]; b) Ranking and/or outranking procedures [14]-[22]; c) Fuzzy set theory and conjoint analysis [23]-[28]; d) Determination of decision maker's aspiration levels and objective weights [29] [30] [31] [32].

Some researchers have focused on the use of convex cones for solving the discrete alternative MCDM problems and investigating preference structures. Nasrabadi et al. [33] provide a review of their own research as well as those of a number of other researchers on using convex preference cones in MCDM. Nieto, et al. [34] present a combined life cycle assessment (LCA) and costing analysis model to address certain issues in earth-resource systems. Karakaya et al. [35] investigate a large family of decision maker's preference functions associated with the discrete alternative MCDM problems. They develop the theory and algorithms for achieving the optimum solution for general preference functions and their special cases. The article includes a comprehensive computational study and demonstrates that reaching the optimum requires fewer pairwise comparisons when the shape of the preference function is known in advance. Karsu [36] 
presents a review of the literature on interactive MCDM approaches. The article focuses on studies that use convex cones for representing the DM's preference structure and discusses various assumptions regarding such structures. The article also offers potential areas for future research.

The purpose of this paper is to develop a mathematical framework and the underpinnings for a convergent interactive method for discrete alternative MCDM problems in which some objectives might have indifference regions also called "satisficing thresholds". We draw from the works of Lotfi et al. [30], Koksalan, et al. [2] and Zak and Kruszynski [37]. The contributions of this paper include: a) Extending the previous works by extensively developing a theory for the approach, particularly for MCDM problems having objectives with indifference regions; b) Developing a convergent solution method; c) Investigating the effects of presence of indifference regions on the efficiency of the method.

The structure of the remainder of the paper is as follows. In section two we discuss the problem statement and our approach, and in section three we present the theory of cone-dominance and extend it by presenting three new theorems. In section four we discuss the solution approach and in section five we present a numerical example. Section six consists of the computational experience and we conclude the paper in section seven. Appendix A presents our notations.

\section{Problem Statement and Our Approach}

The problem that we consider is to maximize $g\left(u_{1}\left(z_{1}\right), u_{2}\left(z_{2}\right), \cdots, u_{p}\left(z_{p}\right)\right)$, where $u_{1}\left(z_{1}\right), u_{2}\left(z_{2}\right), \cdots, u_{p}\left(z_{p}\right)$ are the marginal utility functions of the $p$ objectives, and $g$ is the overall utility or value function of $p$ objectives' utility functions. The function $g$ is assumed to be a non-decreasing quasiconcave function of its arguments. Hence, we assume:

$$
\frac{\partial g}{\partial u_{i}} \geq 0 \text { for all } i
$$

and that each $u_{i}\left(Z_{i}\right)$ is in turn a concave function of $Z_{i}$. Each objective $Z_{i}$ can be of one of five types as described below and shown in Figure 1:

1) Objectives that are to be minimized. For such objectives, less is always preferred to more. We call these decreasing objectives.

2) As in 1 above but with the stipulation that for some objectives there may be a threshold below which there is no benefit from further reduction in the objective.

3) Objectives that are to be maximized. For such objectives, more is always preferred to less. We call these increasing objectives.

4) As in 3 above but with the stipulation that for some objectives there may be a threshold above which there is no benefit from further increase in the objective.

5) Objectives for which there is an optimal value or range. We call these target 

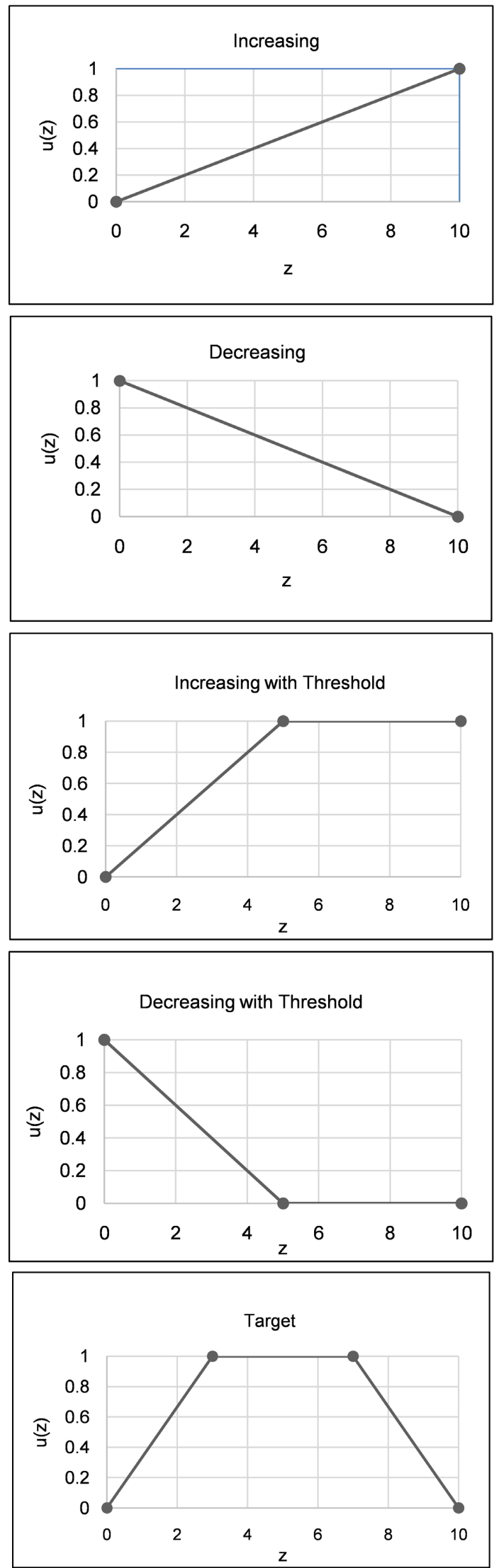

Figure 1. Five types of objectives. 
objectives. An example of such an objective is the distance of one's home from a shopping mall. Most home purchasers would not want it too close, nor too far.

The objectives are assumed to be cardinal in nature, though ordinal objectives may be handled by the method. However, in such a case the procedure becomes a heuristic and cannot guarantee optimality. Also, for each objective the DM may express an absolute minimum and/or an absolute maximum value that is acceptable. These are expressed as constraints that permissible solutions must satisfy.

The proposed method has two phases, finding an initial incumbent and converging to an optimal solution. The initial incumbent can be obtained by finding the nearest non-dominated alternative to the DM's aspiration levels, either obtained directly from the DM or using the median value for each objective. An advantage of using the DM's explicitly stated aspiration levels could be faster convergence to an optimal solution because the initial solution will be closer to his/her most preferred choice. The convergence phase consists of an iterative process whereby the DM responds to questions involving choices between two alternatives, an incumbent solution and a second alternative. The DM's response is then used to eliminate the less preferred alternative. In addition to eliminating the less preferred choice, we use cone-dominance to eliminate additional inferior alternatives within the same iteration. In this case, we use the results of our theoretical developments (Section 3 below) to strengthen the cones, especially for problems that have objectives with indifference regions. Some of the cones in such problems have smaller dimensionality and can eliminate more inferior alternatives within the same iteration.

\section{Theory}

In order to develop a convergent method using cone-dominance, we must first show that the assumed utility function $g$ remains quasi-concave when its arguments are concave functions. These concave functions are the marginal utility functions. The second theoretical development pertains to strengthening the concept of cone-dominance. The strengthening occurs where we have "flat regions" at the maxima of functions $u_{i}$ of $z_{i}$. Such regions occur for target objectives that have lower and upper thresholds specified by the DM as well as increasing and decreasing objectives that have thresholds. We refer to these regions as "indifference regions", and assume the DM considers alternatives with values within these regions to have the same utility values. The strengthening of cones occurs for an objective without an indifference region but one in which an alternative (considered less preferred in a pairwise comparison) has a score equal to the ideal value for that objective. This phenomenon is illustrated in the numerical example below.

We can express the target objectives, increasing with threshold, and decreasing with threshold in terms of their utility functions as follows: 
a) Target Objective:

$$
u_{i}\left(z_{i}^{k}\right)=\left\{\begin{array}{lc}
u_{i 1}\left(z_{i}^{k}\right) & z_{i}^{k}<l t_{i} \\
-u_{i 2}\left(z_{i}^{k}\right) & z_{i}^{k}>u t_{i} \\
u_{i 1}\left(l t_{i}\right)=u_{i 2}\left(u t_{i}\right) & l t_{i} \leq z_{i}^{k} \leq u t_{i}
\end{array}\right.
$$

where $I t_{i}$ and $u t_{i}$ are the lower and upper thresholds for objective $i$ respectively, and $u_{i 1}\left(z_{i}\right)$ and $u_{i 2}\left(z_{i}\right)$ are the increasing and decreasing sides of the objective, respectively, assumed continuous and concave functions of $z_{i}$.

b) Increasing objective with threshold:

$$
u_{i}\left(z_{i}^{k}\right)= \begin{cases}u_{i 1}\left(z_{i}^{k}\right) & z_{i}^{k} \leq u t_{i} \\ u_{i 1}\left(u t_{i}\right) & z_{i}^{k}>u t_{i}\end{cases}
$$

where $u t_{i}$ is the upper threshold for objective $i$ and $u_{i 1}\left(z_{i}\right)$ is a concave function of $Z_{i}$

c) Decreasing objective with threshold:

$$
u_{i}\left(z_{i}^{k}\right)= \begin{cases}-u_{i 2}\left(z_{i}^{k}\right) & z_{i}^{k} \geq l t_{i} \\ -u_{i 2}\left(l t_{i}\right) & z_{i}^{k}<l t_{i}\end{cases}
$$

where $l t_{i}$ is the lower threshold for objective $i$ and $u_{i 2}\left(z_{i}\right)$ is a concave function of $Z_{i}$.

We begin our theoretical development by proving that $g$ remains quasiconcave when its arguments are the marginal utility functions $u$ 's.

Theorem 1. Let $g$ be a quasiconcave and non-decreasing function of a vector $u$, and $u_{i}$ (ith component of $u$ ) be a concave function of $z_{i}$ Then $g$ is a quasiconcave function of the vector $z$ whose components are $z_{i}$.

Proof. Consider two vectors of $z$, namely, $v$ and $w$. Consider the function $g\left(u_{1}\left(z_{1}\right), u_{2}\left(z_{2}\right), \cdots, u_{p}\left(z_{p}\right)\right)$ and a convex combination of $v$ and $w$, $\lambda(v)+(1-\lambda)(w)$. We may then write $g\left(u_{1}\left(\lambda\left(v_{1}\right)+(1-\lambda)\left(w_{1}\right)\right), u_{2}\left(\lambda\left(v_{2}\right)+(1-\lambda)\left(w_{2}\right)\right), \cdots, u_{p}\left(\lambda\left(v_{p}\right)+(1-\lambda)\left(w_{p}\right)\right)\right)$.

By concavity of $u$, we have:

$$
u_{i}\left(\lambda\left(v_{i}\right)+(1-\lambda)\left(w_{i}\right)\right) \geq \lambda u_{i}\left(v_{i}\right)+(1-\lambda) u_{i}\left(w_{i}\right) \text { for all } i .
$$

Further, $g$ is increasing and we have,

$g\left(u_{1}\left(\lambda\left(v_{1}\right)+(1-\lambda)\left(w_{1}\right)\right), u_{2}\left(\lambda\left(v_{2}\right)+(1-\lambda)\left(w_{2}\right)\right), \cdots, u_{p}\left(\lambda\left(v_{p}\right)+(1-\lambda)\left(w_{p}\right)\right)\right)$

$\geq g\left(\lambda u_{1}\left(v_{1}\right)+(1-\lambda) u_{1}\left(w_{1}\right), \lambda u_{2}\left(v_{2}\right)+(1-\lambda) u_{2}\left(w_{2}\right), \cdots, \lambda u_{p}\left(v_{p}\right)+(1-\lambda) u_{p}\left(w_{p}\right)\right)$.

Finally, because $g$ is quasiconcave, $g(\lambda \alpha+(1-\lambda) \beta) \geq \min \{g(\alpha), g(\beta)\}$, hence

$$
\begin{aligned}
& g\left(\lambda u_{1}\left(v_{1}\right)+(1-\lambda) u_{1}\left(w_{1}\right), \lambda u_{2}\left(v_{2}\right)+(1-\lambda) u_{2}\left(w_{2}\right), \cdots, \lambda u_{p}\left(v_{p}\right)+(1-\lambda) u_{p}\left(w_{p}\right)\right) \\
& \geq \min \{g(u(v)), g(u(w))\}
\end{aligned}
$$


The next two lemmas are extensions of Lemma 1 and Lemma 2 by Korhonen et al. [3]. The lemmas facilitate clarity of presentation and allow us to strengthen the concept of cone-dominance.

Lemma 1. Consider a quasiconcave function $g(u)$ as in Theorem 1 defined on the $p$-dimensional Euclidean space $R^{p}$ of real vectors. Let $z^{k} \in R^{p}, k=1, \cdots, m$, be the set of alternatives, and the convex polyhedron spanned by the alternatives $Z=\left\{z \mid \sum \mu_{k} x^{k}, \sum \mu_{k}=1, \mu_{k} \geq 0\right\}$. Further, assume that $g\left(u_{1}\left(z_{1}^{k}\right), u_{2}\left(z_{2}^{k}\right), \cdots, u_{p}\left(z_{p}^{k}\right)\right)=\min _{j} g\left(u_{1}\left(z_{1}^{j}\right), u_{2}\left(z_{2}^{j}\right), \cdots, u_{p}\left(z_{p}^{j}\right)\right)$. If $y \in Z$, it follows that $g(u(y)) \geq g\left(u\left(z^{k}\right)\right)$.

Proof. By Theorem 1, $g$ retains its quasiconcavity when defined on $z$. The remainder of the proof is similar to that of Lemma 1 by Korhonen et al. (1984) [3] and will not be reiterated.

Lemma 2. Let $g(u)$ be as in Lemma 1, consider distinct points $z^{k} \in R^{p}$, $k=1,2, \cdots, m$, and assume $g\left(u\left(z^{k}\right)\right)<g\left(u\left(z^{j}\right)\right), j \neq k$. If $x \in X$ and $x \neq z^{k}$, where $X=\left\{x \mid x=z^{k}+\sum_{j \neq k} \mu_{j}\left(z^{k}-z^{j}\right), \mu_{j} \geq 0\right\}$ it follows that $g(u(x)) \leq g\left(u\left(z^{k}\right)\right)$.

Proof. By Theorem 1, $g$ is quasiconcave on $z$. The rest of the proof is similar to that of Lemma 2 by Korhonen et al. [3] and will not be reiterated. $\square$

The following theorem is an extension of Theorem 1 of Korhonen et al. [3].

Theorem 2. Let $g$ be quasiconcave and non-decreasing on $R^{p}$. Consider distinct points $z^{j} \in R^{p}, j=1,2, \cdots, m$, and any point $y \in R^{p}$. Further, assume that $g\left(u\left(z^{k}\right)\right)<g\left(u\left(z^{j}\right)\right), j \neq k$. Then, if $\varepsilon \geq 0$ in the following linear program,

$\max \varepsilon$

subject to : $\sum_{j \neq k} \mu_{j}\left(z^{k}-z^{j}\right)-\varepsilon \geq y-z^{k}, \mu_{j} \geq 0$

it follows that $g\left(u\left(z^{k}\right)\right) \geq g(u(y))$.

Korhonen et al. [3] show that one can construct a cone, originating at $Z^{k}$, using a linear combination of the remaining $m-1$ points (other than $k$ ). Any point $y$ satisfying Theorem 2 is dominated by at least one point in the cone and may be eliminated from further analysis.

The following theorem provides additional properties for constructing stronger cones. The theorem shows that for a less preferred alternative, if an objective has a score which attains maximum utility, the resulting cone is of smaller dimension and is therefore stronger.

Theorem 3. Let $g$ be quasiconcave and non-decreasing on $R^{p}$. Consider distinct points $z^{j} \in R^{p}, \quad j=1,2, \cdots, m$, and any point $y \in R^{p}$ dominated by $z^{k}$ as in Theorem 2. Further, assume that $g\left(u\left(z^{k}\right)\right)<g\left(u\left(z^{j}\right)\right), j \neq k$ and $u_{t}\left(z_{t}^{k}\right)=\max _{q} u_{t}\left(z_{t}^{q}\right)$, then the following linear program is consistent, $\max \varepsilon$

subject to : $\mu\left(z_{i}^{k}-z_{i}^{j}\right)-\varepsilon \geq y_{i}-z_{i}^{k}, i=1,2, \cdots, p, i \neq t$

$$
\mu \geq 0, \varepsilon \geq 0
$$


Proof. Let $\mu=\mu^{0}$ be the solution to the linear program in Theorem 2. From Theorem 2 it follows that,

$$
z_{i}^{k}+\mu^{0}\left(z_{i}^{k}-z_{i}^{j}\right) \geq y_{i}, i=1,2, \cdots, p, i \neq t
$$

Further, $z_{t}^{k} \geq z_{t}^{j}$ (since $\left.u\left(z_{t}^{k}\right)=\max _{q} u\left(z_{t}^{q}\right)\right)$ which implies $z_{t}^{k}-z_{t}^{j} \geq 0$, hence $z_{t}^{k}+\mu^{0}\left(z_{t}^{k}-z_{t}^{j}\right) \geq z_{t}^{k}$. Because $u_{t}($.$) is assumed concave z_{t}^{k} \geq y_{t}$. It follows that $z_{t}^{k}+\mu^{0}\left(z_{t}^{k}-z_{t}^{j}\right) \geq y_{t}$.

Theorem 3 is similar to Theorem 2 for checking whether an alternative $y$ is cone-dominated but has stronger properties. If one or more of the objectives of the less preferred alternative in the pairwise comparisons attain maximum value or are within the indifference regions, such objectives need not be included in the linear program or checking for dominance of the remaining alternatives.

\section{The Method}

The method consists of two phases. The first phase involves selecting the initial solution (incumbent) and second phase is the iterative process to arrive at the optimal solution (convergence).

\subsection{Phase I-The Initial Incumbent}

The method of generating an initial incumbent draws from the Aspiration-Based Interactive Method (AIM) of Lotfi, et al. [30]. The AIM method uses a scalarizing function proposed by Wierzbicki [29], with a weight on objective $i$ given by $\left(A_{i}-N_{i}\right) /\left(I_{i}-N_{i}\right)$, where $A_{i}, I_{i}$, and $N_{i}$ are the aspiration level, ideal value, and nadir value for objective $i$, respectively. The weights are valid for both maximization and minimization objectives. They are chosen to reflect the increasing importance attached to objective $i$ as the aspiration levels are closer to the ideal values. The ideal and nadir values are defined as follows:

$$
\begin{gathered}
I_{i}= \begin{cases}\min \left[u t_{i}, \max _{k}\left\{z_{i}^{k}\right\}\right] & \text { if } i \text { is maximizing } \\
\max \left[l t_{i}, \min _{k}\left\{z_{i}^{k}\right\}\right] & \text { if } i \text { is minimizing }\end{cases} \\
N_{i}= \begin{cases}\min \left\{z_{i}^{k}\right\} & \text { if } i \text { is maximizing } \\
\max \left\{z_{i}^{k}\right\} & \text { if } i \text { is minimizing }\end{cases}
\end{gathered}
$$

We assume $I_{i} \neq N_{i} \quad \forall i$, otherwise objective $i$ with $I_{i}=N_{i}$ is a "must" criterion. All alternatives with $z_{i}^{k} \neq I_{i}$ are removed from further consideration. Also, objective $i$ is removed and the ideal and nadir values are updated.

There are two options for obtaining the aspiration levels. The DM may wish to express the aspiration levels explicitly in which case they will be used to solve P1 below. Alternatively, they may be set to the median value for each objective as follows.

Define: 


$$
\begin{aligned}
& z_{i}^{\prime k}= \begin{cases}z_{i}^{k} & \text { if } z_{i}^{k}<I_{i} \text { and } i \text { is maximizing } \\
I_{i} & \text { if } z_{i}^{k} \geq I_{i} \text { and } i \text { is maximizing }\end{cases} \\
& z_{i}^{\prime k}= \begin{cases}z_{i}^{k} & \text { if } z_{i}^{k}>I_{i} \text { and } i \text { is minimizing } \\
I_{i} & \text { if } z_{i}^{k} \leq I_{i} \text { and } i \text { is minimizing }\end{cases}
\end{aligned}
$$

We must have at least two distinct alternatives $k$ and $j$ with $z_{i}^{\prime k} \neq z_{i}^{\prime j}$ because $I_{i} \neq N_{i} \quad \forall i$. Let $z_{i}^{\prime[k]}$ be an ordered set of $z_{i}^{\prime k}$ such that: $z_{i}^{\prime[1]}<z_{i}^{\prime[2]}<\cdots<z_{i}^{\prime\left[m_{i}\right]}$, where $m_{i}$ is the number of distinct values for objective $i$ between $N_{i}$ and $I_{i}$ (inclusive). The aspiration levels are set at:

$$
A_{i}=\left\{\begin{aligned}
z_{i}^{\prime}\left[\left\lfloor\frac{m_{i}+1}{2}\right]\right] & \text { if } i \text { is maximizing } \\
z_{i}^{\prime \prime}\left[\left\lceil\frac{m_{i}+1}{2}\right\rceil\right] & \text { if } i \text { is minimizing }
\end{aligned}\right.
$$

The initial incumbent is the nearest non-dominated alternative to the aspiration levels using a Chebycheff distance function. It is obtained by solving the following problem:

$$
\text { (P1) } \min _{k} \max _{i}\left\{d_{i}\right\}+\varepsilon \sum_{i} d_{i}
$$

where,

$$
d_{i}=\left(A_{i}-N_{i}\right)\left(A_{i}-z_{i}^{k}\right) /\left(I_{i}-N_{i}\right)^{2}, \text { and } \varepsilon>0 \text { but small, }
$$

and $A_{i}-N_{i}$ is the difference between the aspiration level $A_{i}$ for objective $i$ and the nadir value $N_{i}$ for objective $i ; A_{i}-z_{i}^{k}$ is the difference between the aspiration level $A_{i}$ and the value of alternative $k$ on objective $i$; and $I_{i}-N_{i}$ is the difference between the ideal value and nadir value for objective $i$. The solution to $\mathrm{P} 1$ is used as the starting alternative or incumbent for the convergence phase. In this study, we set the aspiration levels to the median value for each objective.

\subsection{Phase II-Convergence}

In this phase we present an algorithm for assuring convergence to an optimal solution. A pairwise comparison approach is used whereby the DM is presented with the incumbent solution and another alternative and asked to select the preferred choice. The preferred then choice becomes the incumbent, the lesser preferred choice is eliminated, and the process is repeated. If there are $\mathrm{m}$ alternatives, through $m-1$ comparisons we can surely know the most preferred solution. Using the mathematical properties developed in Section 3, we may be able to substantially reduce the number of pairwise comparisons required. The way in which alternatives may be eliminated is by showing cone-dominance of certain alternatives about which questions are never asked. We can significantly strengthen the concept of cone-dominance here because of the particular nature of our problem, namely the existence of indifference regions within different types of criteria.

To determine the second alternative to use for pairwise comparisons, one approach is to choose adjacent efficient solutions to the current incumbent solu- 
tion. While this approach has been shown to be promising [38], it requires solving several linear programming problems of special structures. Another approach is to capitalize on the "neighboring" concept of ELECTRE by Roy [14] [15] and select an outranking solution for comparison with the current solution. Roy's outranking method is relatively simpler to implement and will be used in our study. When there is more than one outranking solution to the current incumbent, we choose the one for which most of the objectives have their values in the most preferred range. The intention is that such a solution will be less preferred than the incumbent, and that the strong cone resulting from such a preference will eliminate more of the remaining alternatives. The convergence phase is described below.

Let alternative $j$ be the initial incumbent solution, define $M=\{1,2, \cdots, m\}$ as the index set of alternatives. Determine $s(k)=\left\{i \mid z_{i}^{\prime k}=I_{i}\right\}$ for all $k \in M, s(k)$ is the index set of the objectives, for alternative $k$, with values equal to the ideal point (for that objective).

Step 1. Determine the set of out-ranking alternatives $B_{j}$ (see Appendix B) to the current incumbent solution $j$. If $\left|B_{j}\right|=\varnothing$, select one of the remaining alternatives at random.

Step 2. Find $l \in B_{j}$ with $|s(l)|=\max _{k \in B_{j}}\{|s(k)|\}$. Present the two alternatives $l$ and $j$ to DM and ask DM to choose one. If $l$ is preferred to $j$ go to Step 3 , otherwise go to Step 4.

Step 3. Let $v \leftarrow z^{\prime l}$ and $w \leftarrow z^{\prime j}$, then let $M \leftarrow M \backslash\{j\}$ and $j \leftarrow l$. Go to step 5.

Step 4. Let $v \leftarrow z^{\prime j}$ and $w \leftarrow z^{\prime l}$, then let $M \leftarrow M \backslash\{l\}$ and $B_{j} \leftarrow B_{j} \backslash\{l\}$.

Step 5. Form the linear program,

$$
\begin{aligned}
& \max \varepsilon \\
& \text { s.t. } w_{i}+\mu_{i}\left(w_{i}-v_{i}\right) \geq z_{i}^{\prime k}+\varepsilon, i \notin s(k), k \in M
\end{aligned}
$$

If $\varepsilon \geq 0$, then alternative $k$ is cone-dominated. Let $M \leftarrow M \backslash\{k\}$. If $|M|=1$, then stop, alternative $j$ is the optimal solution. Otherwise, repeat Step 5 for all $k \in M$ then go to Step 1 .

\section{A Numerical Example}

In this section, we present a numerical example to illustrate the convergence phase in some detail. The example illustrates the application of the method in finance. More specifically, suppose a decision maker (DM) wishes to choose a stock (an equity) from those listed on NASDAQ ${ }^{\mathrm{rm}}$ and $\mathrm{NYSE}^{\mathrm{Tm}}$. The DM wishes to use the following five objectives:

1-Price: Stock closing price to be minimized;

2-Earning per share (EPS): To be maximized with absolute minimum of 5.0;

3 -Price to earnings ratio (PE): To be minimized with absolute maximum of 20 ; 
4-Price to book value (PB): To be minimized with absolute maximum of 2.0;

5-Dividend percent per share (DIV): To be maximized.

The above rules were applied to the stocks listed on the stated markets on June 20, 2021. Ninety-seven stocks met the requirements. We selected a random sample of 20 (see Table 1) stocks from the 97 stocks to illustrate the method.

Assume that the DM's implicit marginal utility functions for the five stated objectives are linear and consist of $u_{j}\left(z_{j}^{k}\right)=\left(z_{j}^{k}-N_{j}\right) /\left(I_{j}-N_{j}\right), j=1, \cdots, 5$, and that the DM has assigned a threshold of 14.0 for PE and 4.0 for DIV (i.e., It $_{3}$ $=14.0$ and $\left.u t_{5}=4.0\right)$. We arbitrarily assume that the DM's most preferred stock is FNB, and that his implicit aggregate utility function $g($.$) is the concave func-$ tion $-0.25\left(u_{1}\left(z_{1}\right)-0.863\right)^{2}-0.20\left(u_{2}\left(z_{2}\right)-0.119\right)^{2}-0.15\left(u_{3}\left(z_{3}\right)-0.148\right)^{2}-0.15$ $\left(u_{4}\left(z_{4}\right)-0.920\right)^{2}-0.25\left(u_{5}\left(z_{5}\right)-0.309\right)^{2}$, where $u_{j} s$ are the marginal utility functions. The knowledge of this utility function will be used only in answering the yes or no pairwise comparison questions. The marginal utility values and the aggregate utilities are presented in Table 1 . As mentioned, alternative FNB is the

Table 1. Alternative scores and utility values.

\begin{tabular}{|c|c|c|c|c|c|c|c|c|c|c|c|}
\hline \multirow{2}{*}{ Stock } & \multicolumn{5}{|c|}{ Raw Scores } & \multicolumn{5}{|c|}{ Marginal Utility Values } & \multirow{2}{*}{$\begin{array}{l}\text { Aggregate } \\
\text { utility }(g)\end{array}$} \\
\hline & Price & EPS & $\mathrm{PE}$ & $\mathrm{PB}$ & DIV & $u_{1}\left(z_{1}\right)$ & $u_{2}\left(z_{2}\right)$ & $u_{3}\left(z_{3}\right)$ & $u_{4}\left(z_{4}\right)$ & $u_{5}\left(z_{5}\right)$ & \\
\hline AGM & 61.89 & 11.38 & 9.19 & 1.43 & 2.31 & 0.066 & 0.045 & 0.499 & 0.534 & 0.058 & -0.217 \\
\hline AMRK & 15.86 & 80.00 & 14.22 & 1.62 & 2.02 & 0.823 & 0.572 & 0.480 & 0.352 & 0.000 & -0.130 \\
\hline AXS & 65.89 & 127.45 & 13.85 & 1.11 & 2.29 & 0.000 & 0.937 & 0.499 & 0.840 & 0.054 & -0.356 \\
\hline BCRH & 19.30 & 109.09 & 12.54 & 0.94 & 6.25 & 0.766 & 0.795 & 0.499 & 1.000 & 0.395 & -0.115 \\
\hline BHLB & 35.80 & 7.41 & 19.83 & 1.16 & 2.35 & 0.495 & 0.014 & 0.000 & 0.786 & 0.066 & -0.057 \\
\hline BNS & 56.62 & 5.81 & 12.18 & 1.67 & 3.97 & 0.152 & 0.002 & 0.499 & 0.306 & 0.389 & -0.206 \\
\hline BSMX & 9.24 & 21.43 & 14.61 & 1.94 & 7.03 & 0.932 & 0.122 & 0.446 & 0.056 & 0.395 & -0.128 \\
\hline BSRR & 24.80 & 12.90 & 18.58 & 1.60 & 2.30 & 0.676 & 0.056 & 0.107 & 0.375 & 0.056 & -0.070 \\
\hline $\mathrm{FFBC}$ & 25.45 & 5.56 & 16.87 & 1.79 & 2.69 & 0.665 & 0.000 & 0.254 & 0.198 & 0.134 & -0.100 \\
\hline $\mathrm{FNB}^{\star}$ & 13.44 & 21.05 & 18.10 & 1.02 & 3.57 & 0.863 & 0.119 & 0.148 & 0.920 & 0.309 & 0.000 \\
\hline GLW & 29.88 & 8.11 & 8.14 & 1.71 & 2.09 & 0.592 & 0.020 & 0.499 & 0.274 & 0.014 & -0.123 \\
\hline LCNB & 19.95 & 6.90 & 15.28 & 1.35 & 3.27 & 0.756 & 0.010 & 0.390 & 0.609 & 0.250 & -0.029 \\
\hline MFC & 17.30 & 37.50 & 15.25 & 1.19 & 3.40 & 0.799 & 0.245 & 0.392 & 0.762 & 0.275 & -0.017 \\
\hline ORI & 19.95 & 5.88 & 12.85 & 1.14 & 3.81 & 0.756 & 0.002 & 0.499 & 0.804 & 0.357 & -0.027 \\
\hline PAG & 42.63 & 9.01 & 10.61 & 2.00 & 2.87 & 0.383 & 0.027 & 0.499 & 0.000 & 0.170 & -0.210 \\
\hline PBF & 19.97 & 135.71 & 11.51 & 1.12 & 5.97 & 0.755 & 1.000 & 0.499 & 0.826 & 0.395 & -0.180 \\
\hline PIR & 5.10 & 28.57 & 13.86 & 1.47 & 5.43 & 1.000 & 0.177 & 0.499 & 0.497 & 0.395 & -0.053 \\
\hline STBA & 34.36 & 14.29 & 16.18 & 1.39 & 2.35 & 0.519 & 0.067 & 0.312 & 0.570 & 0.066 & -0.067 \\
\hline $\mathrm{TD}$ & 47.81 & 6.30 & 12.78 & 1.70 & 3.71 & 0.297 & 0.006 & 0.499 & 0.277 & 0.337 & -0.163 \\
\hline UMPQ & 17.19 & 8.00 & 16.47 & 0.97 & 3.71 & 0.801 & 0.019 & 0.287 & 0.971 & 0.337 & -0.006 \\
\hline
\end{tabular}

${ }^{*}$ Assumed optimal alternative. 
optimum with aggregate utility value of zero.

The median values are $19.97,12.90,13.86,1.39$, and 3.40 , for objectives one through five, respectively. The closest non-dominated solution to the medians is $\mathrm{BCRH}$ which will be used as the initial incumbent. The closest outranking solutions to BCRH is PBF. We then ask the DM to compare the two stocks PBF and BCRH. Based on the assumed DM's utility function, alternative BCRH is preferred to PBF. This information is utilized to form the first cone. Because the PE, $\mathrm{PB}$ and DIV criteria of the preferred alternative (BCRH) are of maximum utility, the resulting cone will be two dimensional and will not include the $\mathrm{PE}, \mathrm{PB}$ and DIV. That is,

$$
\begin{array}{cc}
\text { PBF } & \text { PBF } \quad \text { BCRH } \\
{\left[\begin{array}{c}
19.97 \\
135.71
\end{array}\right]+\mu\left[\begin{array}{c}
19.97-19.30 \\
135.71-109.09
\end{array}\right]}
\end{array}
$$

This cone dominates alternatives AGM, BHLB, BNS, BSRR, FFBC, GLW, PAG, STBA, and TD. These alternatives, together with alternative PBF are dropped from further consideration. Next, the DM is asked to compare BCRH and MFC. Because $u(\mathrm{BCRH})=-0.115$ is smaller than $u(\mathrm{MFC})=-0.017$, alternative MFC is preferred to BCRH. The resulting cone (not shown) dominates alternatives AXS, LCNB, and ORI. These alternatives together with BCRH are dropped from further consideration. Next, we compare MFC with FNB. Alternative FNB is preferred, causing to drop MFC. Alternative FNB is compared with PIR with FNB being preferred and the resulting cone dominates alternative BSMX. Alternatives PIR and BSMX are dropped from further consideration. FNB is then compared with AMRK, dropping AMRK. Finally, FNB is compared with the only remaining alternative UMPQ, FNB being the preferred alternative and the procedure stops with FNB as the optimal solution. In total, the algorithm converges to the optimum by asking six pairwise comparisons instead of 19 questions. Moreover, if we would have included thresholds for the other three criteria (Price, EPS, and PB) the algorithm might have converged even faster.

\section{Computational Experience}

We evaluated the performance of the method by solving several hundred randomly generated test problems. Of particular interest is the potential impacts of presence of indifference regions on the number of pairwise comparisons required to arrive at the optimal solution. The method was programmed in Visual Basic within the Visual Studio 17 on a desktop computer with an AMD Ryzen 5 1600 six core processor at 3.3 giga hertz, having 16.0 gig of RAM. The study design parameters were as follows:

- Four levels of alternatives $(m): 10,20,50$ and 100;

- Four levels of objectives $(p): 4,6,8$ and 10;

- Four levels of number of objectives with indifference regions: none, $25 \%$ of objectives, $50 \%$ of objectives, and $100 \%$ of objectives;

- Three levels of indifference regions (for objectives with indifference regions): 
$25 \%, 50 \%, 75 \%$;

- Five repetitions per cell.

All of the objectives were assumed increasing. The DM's responses were simulated using a quadratic utility function: $\max -\sum w_{i}\left(u_{i}\left(z_{i}^{*}\right)-u_{i}\left(z_{i}^{k}\right)\right)^{2}$, where $w_{i} s$ were Uniform random variates between 0 and 1, rescaled to ensure $\sum w_{i}=1$, and $z^{*}$ was the optimal alternative, selected at random from among the set of alternatives for that problem. The alternative scores were uniformly distributed between 0 and 20 and the marginal utility function $u_{i}\left(z_{i}\right)$ was assumed to be $u_{i}\left(z_{i}\right)=\max \left\{\left(z_{i}-N_{i}\right) /\left(I_{i}-N_{i}\right), 1\right\}$. These characteristics resulted in 800 test problems. The performance measure was the number of pairwise comparisons (questions to be asked of DM) required to arrive at the optimal solution. The computational times were insignificant, amounting to no more than a fraction of a second to solve each problem. Therefore, they were not recorded.

Table 2 present the results of the random test problems. Cell entries are the

Table 2. Results of convergence phase.

\begin{tabular}{|c|c|c|c|c|c|c|c|c|c|c|c|}
\hline \multirow{3}{*}{\multicolumn{2}{|c|}{ Level of Threshold }} & \multicolumn{10}{|c|}{ Percent of Objectives with Threshold } \\
\hline & & \multirow{2}{*}{$\begin{array}{c}0 \\
\text { N/A }\end{array}$} & \multicolumn{3}{|c|}{$25 \%$} & \multicolumn{3}{|c|}{$50 \%$} & \multicolumn{3}{|c|}{$100 \%$} \\
\hline & & & $25 \%$ & $50 \%$ & $75 \%$ & $25 \%$ & $50 \%$ & $75 \%$ & $25 \%$ & $50 \%$ & $75 \%$ \\
\hline M & $\mathrm{p}$ & & & & & & & & & & \\
\hline \multirow[t]{5}{*}{10} & 4 & $4.8^{*}$ & 5.0 & 6.2 & 5.8 & 4.2 & 4.4 & 5.6 & 1.8 & 4.4 & 3.6 \\
\hline & 6 & 6.6 & 6.6 & 7.4 & 7.2 & 5.2 & 7.6 & 6.4 & 5.0 & 7.0 & 7.0 \\
\hline & 8 & 8.6 & 7.4 & 8.6 & 8.8 & 8.2 & 7.8 & 8.0 & 6.8 & 8.0 & 8.2 \\
\hline & 10 & 9.0 & 8.4 & 8.0 & 8.4 & 8.2 & 8.6 & 8.6 & 4.4 & 8.4 & 8.8 \\
\hline & Mean & 7.3 & 6.9 & 7.6 & 7.6 & 6.5 & 7.1 & 7.2 & 4.5 & 7.0 & 6.9 \\
\hline \multirow[t]{5}{*}{20} & 4 & 7.0 & 7.8 & 7.2 & 7.8 & 6.0 & 6.6 & 8.0 & 1.6 & 6.8 & 8.2 \\
\hline & 6 & 11.4 & 11.2 & 11.0 & 14.6 & 10.4 & 12.2 & 10.8 & 4.6 & 9.4 & 12.4 \\
\hline & 8 & 15.2 & 13.0 & 14.0 & 16.6 & 10.2 & 15.6 & 14.4 & 8.2 & 14.8 & 15.6 \\
\hline & 10 & 18.2 & 16.4 & 18.4 & 18.0 & 15.6 & 15.4 & 18.0 & 15.2 & 17.6 & 18.0 \\
\hline & Mean & 13.0 & 12.1 & 12.7 & 14.3 & 10.6 & 12.5 & 12.8 & 7.4 & 12.2 & 13.6 \\
\hline \multirow[t]{5}{*}{50} & 4 & 11.2 & 9.2 & 13.8 & 9.6 & 4.2 & 9.4 & 8.2 & 2.0 & 3.8 & 10.8 \\
\hline & 6 & 22.8 & 15.4 & 20.6 & 21.0 & 15.0 & 15.6 & 24.4 & 2.8 & 14.8 & 21.2 \\
\hline & 8 & 37.2 & 33.0 & 29.6 & 40.0 & 17.2 & 28.0 & 36.0 & 10.2 & 20.2 & 33.4 \\
\hline & 10 & 38.4 & 44.0 & 34.2 & 43.2 & 29.8 & 38.0 & 43.8 & 8.8 & 32.6 & 43.8 \\
\hline & Mean & 27.4 & 25.4 & 24.6 & 28.5 & 16.6 & 22.8 & 28.1 & 6.0 & 17.9 & 27.3 \\
\hline \multirow[t]{5}{*}{100} & 4 & 11.0 & 9.4 & 11.2 & 13.4 & 8.8 & 8.8 & 17.0 & 1.4 & 4.6 & 13.6 \\
\hline & 6 & 37.2 & 25.4 & 32.6 & 36.0 & 15.2 & 28.6 & 46.6 & 2.0 & 21.0 & 37.2 \\
\hline & 8 & 59.8 & 51.6 & 62.4 & 63.8 & 35.4 & 49.4 & 57.2 & 3.0 & 32.4 & 59.6 \\
\hline & 10 & 75.0 & 68.4 & 71.2 & 77.6 & 61.2 & 49.8 & 83.2 & 9.6 & 65.4 & 78.6 \\
\hline & Mean & 45.8 & 38.7 & 44.4 & 47.7 & 30.2 & 34.2 & 51.0 & 4.0 & 30.9 & 47.3 \\
\hline
\end{tabular}

${ }^{\star}$ Cell entries are average number of pairwise comparisons. 
average number of pairwise comparisons (comparisons) to reach the optimum over the five repetitions in that cell. We can observe that in general, our method significantly reduces the number of comparisons required to arrive at the optimal solution. For instance, even with no indifference region ((Table 2) column with Percent of objectives with threshold heading 0 ) for the problem set with $m$ $=50$ and $p=4$ the average number of comparisons is 11.2 rather than $m-1=$ 49 , or a reduction of about $77 \%$. For the problem set with $p=10$ and the same number of alternatives, the average increases to 38.4 , still a saving of about $22 \%$. Naturally, as the problem size increases both with respect to the number of objectives and the number of alternatives, the number of comparisons needed to reach the optimum increases.

The presence of indifference regions significantly impacts the number of comparisons required to arrive at the optimum. As the size of the indifference region increases, the number of comparisons decreases. Consider for instance problem sets with just $25 \%$ of the objectives having indifference regions (Figure 2 ). For problems with $m=100$, the average number of comparisons for problems with $25 \%$ threshold level (widest indifference region) is 38.7 , compared with 47.7 for problems at $75 \%$ threshold level (the narrowest indifference region). Similarly, as the percentage of objectives with indifference regions increases, the number of comparisons decreases. For problem sets with $m=100$, the average number of comparisons (over all threshold levels) for problems with $25 \%$ of objectives having indifference regions is $(38.7+444.4+47.7) / 3=43.6$, versus 38.4 for those with $50 \%$ of objectives and 27.4 for those with $100 \%$ of objectives having indifference regions. The reason is that for a given objective, more of the alternatives will have the same utility value, increasing the chances of more alternatives being cone-dominated.

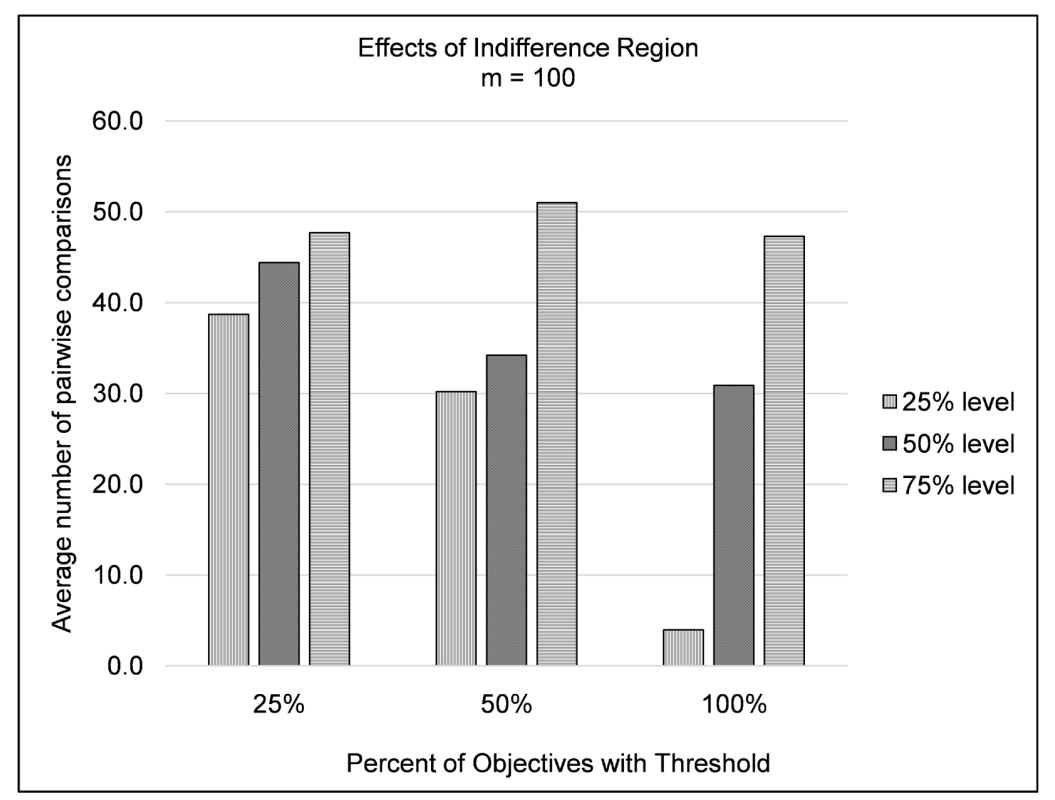

Figure 2. Effects of indifference region on the number of pairwise comparisons. 


\section{Conclusion}

In this paper, we have significantly extended the theory of cone-dominance to address the discrete alternative MCDM problems that have objectives with indifference regions. We provided theory and then developed a method, based on using pairwise comparisons of the alternatives by the DM to identify the most preferred choice. A numerical example was provided, illustrating an application of the method in finance. A simulation study, assessing the performance of the method on several hundred randomly generated problems was presented. The proposed method appears to be promising as it can be used to reduce the number of pairwise comparisons required to arrive at the DM's most preferred solution alternative. Results of the simulation study clearly delineate a significant reduction in the number of such comparisons for objectives having indifference regions. This knowledge can be used to encourage the DM to carefully examine each criterion to identify possible indifference regions thereby reducing the number of questions needed to arrive at the most preferred choice.

\section{Conflicts of Interest}

The author declares no conflicts of interest regarding the publication of this paper.

\section{Funding Source}

This research did not receive any specific grant from funding agencies in the public, commercial, or not-for-profit sectors.

\section{References}

[1] Korhonen, P. (1992) Multiple Criteria Decision Support: The State of Research and Future Directions. Computers \& Operations Research, 19, 549-551. https://doi.org/10.1016/0305-0548(92)90024-Y

[2] Koksalan, M., Karwan, M.H. and Zionts, S. (1984) An Improved Method for Solving Multiple Criteria Problems Involving Discrete Alternatives. IEEE Transactions on Systems, Man, and Cybernetics, 14, 24-34. https://doi.org/10.1109/TSMC.1984.6313266

[3] Korhonen, P., Wallenius, Y. and Zionts, S. (1984) Solving the Discrete Multiple Criteria Problem Using Convex Cones. Management Science, 30, 1336-1345. https://doi.org/10.1287/mnsc.30.11.1336

[4] Ramanathan, R., Ravindran, A. and Mathirajan, M. (2017) Multi-Criteria Decision Making: An Overview and a Comparative Discussion. In: Ramanathan, R., Mathirajan, M. and Ravindran, A., Eds., Big Data Analytics Using Multiple Criteria Decision-making Models, CRC Press, Boca Raton, 22-59. https://doi.org/10.1201/9781315152653-2

[5] Edwards, W. (1977) How to Use Multiattribute Utility Measurement for Social Decision Making. IEEE Transactions on Systems, Man, and Cybernetics, 7, 326-340. https://doi.org/10.1109/TSMC.1977.4309720

[6] Saaty, T.L. (1980) The Analytic Hierarchy Process: Planning, Priority Setting and Resource Allocation. McGraw-Hill, New York. 
[7] Jones, M., Hope, C. and Hughes, R. (1990) A Multi-Attribute Value Model for the Study of UK Energy Policy. Journal of the Operational Research Society, 41, 919-929. https://doi.org/10.1057/jors.1990.144

[8] Von Winterfelt, D. and Edwards, W. (1986) Decision Analysis and Behavioral Research. Cambridge University Press, Boston.

[9] Vargas, L.G. (1990) An Overview of the Analytic Hierarchy Process and Its Applications. European Journal of Operational Research, 48, 2-8. https://doi.org/10.1016/0377-2217(90)90056-H

[10] Keeney, R.L. and Raiffa, H. (1993) Decision with Multiple Objectives: Preference and Value Tradeoffs. Cambridge University Press, New York. https://doi.org/10.1017/CBO9781139174084

[11] Keeney, R.L. and McDaniels, T.L. (1999) Identifying and Structuring Values to Guide Integrated Resource Planning at BC Gas. Operations Research, 47, 651-662. https://doi.org/10.1287/opre.47.5.651

[12] Belton, V. (1999) Multi-Criteria Problem Structuring and Analysis in a Value Theory Framework. In: Gal, T., Stewart, T.J. and Hanne, T., Eds., Multicriteria Decision Making: Advances in MCDM Models, Algorithms, Theory, and Applications, Springer, Boston, 335-366. https://doi.org/10.1007/978-1-4615-5025-9_12

[13] Bordley, R.F. and Kirkwood, C.W. (2004) Multiattribute Preference Analysis with Performance Targets. Operations Research, 52, 823-835.

https://doi.org/10.1287/opre.1030.0093

[14] Roy, B. (1968) Classement et choix en presence de points de vue multiples (la methode ELECTRE). Rev. Informat. Rech. Oplle, 8, 57-75.

https://doi.org/10.1051/ro/196802V100571

[15] Roy, B. (1978) ELECTRE III: Un algorithme de classements fondé sur une représentation floue des préférences en présence de critères multiples. Cahiers du Centre détudes de recherche operationnelle, 20, 3-24.

[16] Brans, J.P. (1982) L'ingénièrie de la décision; Elaboration d'instruments d'aide à la decision, La méthode PROMETHEE. In: Nadeau, R. and Landry, M., Eds., L'aide à la décision: Nature, Instruments et Perspectives d Avenir, Presses de l'Université Laval, Québec, 183-213.

[17] Brans, J.P. and Mareschal, B. (1995) The PROMETHEE VI procedure. How to Differentiate Hard from Soft Multicriteria Problems. Journal of Decision Systems, 4, 213-223. https://doi.org/10.1080/12460125.1995.10511652

[18] Roy, B. (1996) Multicriteria Methodology for Decision Aiding. Springer, Boston. https://doi.org/10.1007/978-1-4757-2500-1

[19] Roy, B. and Vanderpooten, D. (1997) An Overview on "The European School of MCDA: Emergence, Basic Features and Current Works”. European Journal of Operational Research, 99, 26-27. https://doi.org/10.1016/S0377-2217(96)00379-7

[20] Hwang, C.L. and Yoon, K. (1981) Multiple Attribute Decision Making: Methods and Applications. Springer Verlag, Berlin, Heidelberg. https://doi.org/10.1007/978-3-642-48318-9

[21] Bouyssou, D. and Vincke, P.H. (1997) Ranking Alternatives on the Basis of Preference Relations: A Progress Report with Special Emphasis on Outranking Relations. Journal of Multi-Criteria Decision Analysis, 6, 77-85. https://doi.org/10.1002/(SICI)1099-1360(199703)6:2\%3C77::AID-MCDA144\%3E3.0 .CO;2-I

[22] Hanne, T. (1999) Meta Decision Problems in Multiple Criteria Decision Making. In: 
Gal, T., Stewart, T.J. and Hanne, T., Eds., Multicriteria Decision Making: Advances in MCDM Models, Algorithms, Theory, and Applications, Springer, Boston, 147-171. https://doi.org/10.1007/978-1-4615-5025-9_6

[23] Green, P.E. and Srinivasan, V. (1978) Conjoint Analysis in Consumer Research; Issues and Outlook. Journal of Consumer Research, 5, 103-123. https://doi.org/10.1086/208721

[24] Bisdorff, R. (2002) Logical Foundation of multIcriteria Preference Aggregation. In: Bouyssou, D., Jacquet-Lagrèze, E., Perny, P., Słowiński, R., Vanderpooten, D. and Vincke P., Eds., Aiding Decisions with Multiple Criteria, Springer, Boston, 379-403. https://doi.org/10.1007/978-1-4615-0843-4_17

[25] Ammar, S. and Wright, R. (2003) Characteristics and Features of a Performance Evaluation Model Using a Multilevel Fuzzy Rule-Based System. International Journal of Technology, Policy and Management, 3, 301-321. https://doi.org/10.1504/IJTPM.2003.003985

[26] Bouyssou, D. and Pirlot, M. (2002) Nontransitive Decomposable Conjoint Measurement. Journal of Mathematical Psychology, 46, 677-703.

https://doi.org/10.1006/jmps.2002.1419

[27] Sałabun, W. (2015) The Characteristic Objects Method: A New Distance-Based Approach to Multicriteria Decision-Making Problems. Journal of Multi-Criteria Decision Analysis, 22, 37-50. https://doi.org/10.1002/mcda.1525

[28] Hashemi, S.S., Hajiagha, S.H.R., Kazimieras, E. and Mahdiraji, H.A. (2016) Multicriteria Group Decision Making with ELECTRE III Method Based on Interval-Valued Intuitionistic Fuzzy Information. Applied Mathematical Modelling, 40, 1554-1564. https://doi.org/10.1016/j.apm.2015.08.011

[29] Wierzbicki, A.P. (1980) The Use of Reference Objectives in Multiobjective Optimization. In: Fandel, G. and Gal, T, Eds., Multiple Criteria Decision Making Theory and Application, Springer, Berlin, 468-486. https://doi.org/10.1007/978-3-642-48782-8_32

[30] Lotfi, V., Stewart, T. and Zionts, S. (1992) An Aspiration-Level Interactive Model for Multiple Criteria Decision Making. Computers \& Operations Research, 19, 671-681. https://doi.org/10.1016/0305-0548(92)90036-5

[31] Wierzbicki, A.P. (1998) Reference Point Methods in Vector Optimization and Decision Support. Interim Report IR-98-017, International Institute for Applied Systems Analysis, Laxenburg. http://pure.iiasa.ac.at/id/eprint/5631/

[32] Salo, A.A. and Hamalainen, R.P. (2001) Preference Ratios in Multiattribute Evaluation (PRIME)-Elicitation and Decision Procedures under Incomplete Information. IEEE Transactions on Systems, Man, and Cybernetics-Part A: Systems and Humans, 31, 533-545. https://doi.org/10.1109/3468.983411

[33] Nasrabadi, N., Dehnokhalaji, A., Korhonen, P. and Wallenius, J. (2019) Using Convex Preference Cones in Multiple Criteria Decision Making and Related Fields. Journal of Business Economics, 89, 699-717. https://doi.org/10.1007/s11573-019-00935-4

[34] Nieto, A., Bai, Y. and Brownson, J. (2014) Combined Life Cycle Assessment and Costing Analysis Optimization Model Using Multiple Criteria Decision Making in Earth-Resource Systems. Natural Resources, 5, 351-358.

https://doi.org/10.4236/nr.2014.58033

[35] Karakaya G., Koksalan M. and Ahipasaoglu, S.D. (2018) Interactive Algorithms for a Broad Underlying Family of Preference Functions. European Journal of Operational Research, 265, 248-262. https://doi.org/10.1016/j.ejor.2017.07.028 
[36] Karsu O. (2013) Using Holistic Multicriteria Assessments: The Convex Cones Approach. In: Cochran, J., Ed., Wiley Encyclopedia of Operations Research and Management Science, Wiley, New York, 1-14.

https://doi.org/10.1002/9780470400531.eorms1086

[37] Zak, J. and Kruszynski, M. (2015) Application of AHP and ELECTRE III/IV Methods to Multiple Level, Multiple Criteria Evaluation of Urban Transportation Projects. Transportation Research Procedia, 10, 820-830.

https://doi.org/10.1016/j.trpro.2015.09.035

[38] Murat Köksalan, M. and Sagala, P.N.S. (1995) Interactive Approaches for Discrete Alternative Multiple Criteria Decision Making with Monotone Utility Functions. Management Science, 41, 1158-1171. https://doi.org/10.1287/mnsc.41.7.1158. 
Appendix A. Notations

\begin{tabular}{|c|c|}
\hline$m$ & number of decision alternatives \\
\hline$p$ & number of objectives \\
\hline$S$ & set of decision alternatives \\
\hline$Z_{i}$ & objective $i$ \\
\hline$z_{i}^{k}$ & performance measure of alternative $k$ on objective $i$ \\
\hline$u_{i}()$. & marginal utility function for objective $i$ \\
\hline$g()$. & decision maker's utility or value function \\
\hline$l t_{i}$ & lower threshold of objective $i$ \\
\hline$u t_{i}$ & upper threshold of objective $i$ \\
\hline$u_{i 1}\left(z_{i}\right)$ & $\begin{array}{l}\text { marginal utility function for increasing objectives with thresholds } \\
\text { and for increasing side of target objective } i\end{array}$ \\
\hline$u_{i 2}\left(z_{i}\right)$ & $\begin{array}{l}\text { marginal utility function for decreasing objectives with threshold and } \\
\text { for decreasing side of target objective } i\end{array}$ \\
\hline$I_{i}$ & ideal value for objective $i$ \\
\hline$N_{i}$ & nadir value for objective $i$ \\
\hline$A_{i}$ & aspiration value for objective $i$ \\
\hline$z_{i}^{\prime k}$ & modified value of alternative $k$ on objective $i$ \\
\hline$m_{i}$ & number of distinct values for objective $i$ between $N_{i}$ and $I_{p}$ inclusive \\
\hline$M$ & index set of alternatives \\
\hline$w_{i}$ & weight of objective $i$ in the simulated DM preference function \\
\hline$B_{j}$ & set of out-ranking alternatives to alternative $j$ \\
\hline$c$ & $\begin{array}{c}\text { maximum allowable normalized difference for objectives where } \\
\text { alternative } k \text { has better scores than alternative } j\end{array}$ \\
\hline$\alpha_{i}^{k j}$ & $\begin{array}{l}\text { binary function equal } 1 \text { if modified value of alternative } k \text { is greater } \\
\text { than or equal to } j \text { with respect to objective } i\end{array}$ \\
\hline$\beta_{i}^{k j}$ & $\begin{array}{c}\text { difference between normalized values of alternative } j \text { and } k \text { with } \\
\text { respect to objective } i\end{array}$ \\
\hline
\end{tabular}

\section{Appendix B. The Outranking Method}

The following procedure finds other "neighbor" solutions which are in close proximity to the current incumbent, one of which will be used in the pairwise comparisons. To find some neighbor solutions, we use a simplified version of ELECTRE [14]. Alternative $j$ is said to outrank alternative $k$, if and only if the following conditions are satisfied:

(i) the fraction of objectives, for which $j$ is at least as good (any objective value in the indifference region is considered as having the ideal value, and hence is as good as any other) as $k$, is at least $1 / 2$.

(ii) $\max _{i}\left\{\left(z_{i}^{\prime k}-z_{i}^{\prime j}\right) /\left(I_{i}-N_{i}\right)\right\} \leq c$

where $c$ is the maximum allowable value of normalized difference for those objectives which alternative $k$ has better scores than alternative $j$. Intuitively, one 
solution is said to outrank another if it is at least as good as the second one in most respects, and not too much worse in any one respect. In our implementation $c$ was set at $20 \%$. Steps of the procedure are shown below.

Define the binary function $\alpha_{i}^{k, j}$, for alternatives $k$ as:

$$
\alpha_{i}^{k j}=\left\{\begin{array}{cc}
1 & \text { if } z_{i}^{\prime k} \geq z_{i}^{\prime j} \text { and } i \text { is maxiizing, or } \\
& \text { if } z_{i}^{\prime k} \leq z_{i}^{\prime j} \text { and } i \text { is minimizing } \\
0 & \text { otherwise }
\end{array}\right.
$$

Further, let

$$
\beta_{i}^{k j}= \begin{cases}\left(z_{i}^{\prime j}-z_{i}^{\prime k}\right) /\left(I_{i}-N_{i}\right) & \text { if } \alpha_{i}^{k j}=0 \\ -\infty & \text { otherwise }\end{cases}
$$

Initialize $\Gamma \leftarrow \varnothing, B_{j} \leftarrow \varnothing$, and $k \leftarrow 1$.

Step 1. If $\sum_{i} \alpha_{i}^{k, j} \geq\lceil(p+1) / 2\rceil$, let $\Gamma \leftarrow \Gamma \cup\{k\}$.

Step 2. Let $k \leftarrow k+1$. If $k \leq m$, go to Step 1 .

Step 3. If $\Gamma=\varnothing$, stop. There are no outranking solutions to alternative $j$. Otherwise, let $\Gamma^{\prime} \leftarrow \Gamma$, and $k \leftarrow 1$.

Step 4. If $k \notin \Gamma^{\prime}$, go to Step 6 .

Step 5. If $\max _{i}\left\{\beta_{i}^{k, j}\right\}>c$, let $\Gamma^{\prime} \leftarrow \Gamma^{\prime} \backslash\{k\}$.

Step 6. Let $k \leftarrow k+1$. If $k \leq m$, go to Step 4 .

Step 7. If $\Gamma^{\prime}=\varnothing$, stop. There is no remaining outranking solution. Otherwise, let $B_{j} \leftarrow \Gamma^{\prime}$. 